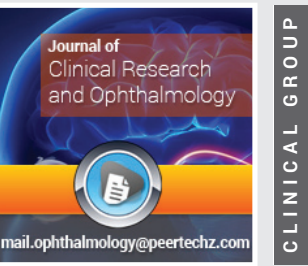

\title{
A case of atypical band
}

\section{shaped keratopathy following intravitreal silicone oil injection in an aphakic eye}

Received: 24 February, 2020

Accepted: 07 April, 2020

Published: 09 April, 2020

*Corresponding author: Dr. Nischala Balakrishna, Junior Resident, Department of Ophthalmology, Vydehi Institute of Medical Sciences and Research Centre, Bangalore, Number 96, First Main Road Seshadripuram, Next To Sri Chaitanya Techno Schools, Bangalore560020, India, Tel: +91 8762558431; 8217352997; E-mail: nischalabalakrishna@gmail.com

Keywords: Silicon oil; Perfluorocarbon; Band keratopathy; Aphakia; Intravitreal

https://www.peertechz.com

\section{Check for updates}

1Junior Resident, Department of Ophthalmology, Vydehi Institute of Medical Sciences and Research

Centre, Bangalore, India

${ }^{2}$ Professor, Department of Ophthalmology, Vydehi Institute of Medical Sciences and Research Centre, Bangalore, India

\section{Abstract}

Introduction: Silicon oil or polydimethylsiloxane fluid is used in complicated retinal detachment surgeries. Although it displays an array of side effects like progressive vascularisation, silicon granules in corneal stroma and corneal decompensation, the lesser known band shaped keratopathy is visually endangering and requires a new management protocol, as was seen in our case.

Case: A 15-year-old girl with vision 6/60 in both eyes diagnosed with bilateral congenital cataract and retinal detachment underwent microincision vitrectomy, lensectomy along with perfluorocarbon and intravitreal silicon oil injection in the right eye under general anaesthesia. Calcific band shaped keratopathy was seen after 8 months which began as fine dust like deposits coalescing to form a horizontal band. A neutral, autoclaved solution of $0.5 \%$ EDTA chelating agent was spread over the cornea and deposits scraped under aseptic precautions after removing the superficial epithelium. A bandage contact lens was placed to enable healing.

Discussion: Calcific band shaped keratopathy with a large silicon oil bubble in anterior chamber commonly seen in aphakics. Calcium salts deposit in the superficial stroma and Bowman's layer due to a lower corneal metabolic rate resulting in less metabolite production. The carbon dioxide evaporation remains unchanged causing increased $\mathrm{pH}$ in superficial layers causing precipitation.

Conclusion: Calcium hydroxyapatite deposition on the cornea is a rare complication of intravitreal silicon oil injection. Aphakics present with this side effect as a result of permanent contact of silicon oil with the corneal endothelium. These side effects must be noticed in order to prevent impending visual decompensation.

\section{Introduction}

Silicone oil or polydimethylsiloxane helps act as an effective internal tamponade in preventing retinal tears and detachments [1]. Its safety profile in vitreoretinal and other posterior segment surgeries has been exceptional especially as an internal tamponade agent. Nevertheless, its secondary anterior segment complications like band shaped keratopathy are visually and cosmetically endangering. Due to the presence of more amounts of retained silicone oil in the anterior chamber, aphakics more commonly present with this complication due to the permanent contact of silicone oil with the corneal endothelium $[2,3]$.

\section{Case description}

A 14-year-old girl diagnosed with bilateral congenital cataract had a vision of $6 / 60$ in both eyes. The intraocular pressure in both eyes was $16 \mathrm{~mm} \mathrm{Hg}$. The anterior segment in both eyes showed cataractous changes. The rest of the anterior segment in both eyes were normal. The posterior segments of both eyes were normal.

Lensectomy was done in the right eye, wherein the lens including the posterior capsule and anterior vitreous was removed. Placement of the intraocular lens was planned at a later date and the right eye was left aphakic. Cataract surgery in the left eye was planned at a later date. 
In cases of bilateral congenital cataracts, the immediate postoperative rehabilitation with correction of aphakia and secondary IOL implantation gives a more predictable refraction postoperatively with minimal complications.

Two years after the surgery, the child returned with complaints of sudden, painless loss of vision in the right eye for one day.

Indirect ophthalmoscopy and B scan revealed a total, funnel shaped rhegmatogenous retinal detachment in the right eye (Figure 1). The left eye was normal.

Anemergencymicroincision vitrectomywith perfluorocarbons and silicone oil (5000 CST) injection under guarded visual prognosis was done under general anaesthesia. As the retinal detachment was very diffuse and not confined to the superior or inferior quadrant, a mixture of polydimethylsiloxane and high-density silicone was used. The post op vision was 1/60 in the right eye.

General physical examination, psychomotor examination and systemic examination were within normal limits. The patient was asked to review every two weeks post-surgery on discharge. Due to the patient's low socio-economic status and lack of resources to travel from her hometown, she was lost to follow-up.

Eight months after the surgery the child returned to our hospital and she had developed a horizontal band shaped keratopathy covering the inferior quadrant of the cornea which encroached the pupillary area. The vision was counting fingers at 2 metres in the right eye (Figure 2 ).

Since the retina was incompletely attached as seen on indirect ophthalmoscopy, a preoperative laser photocoagulation at the site of the detachment was planned to prevent further re-detachment after completely removing the silicon oil.

A neutral, autoclaved solution of $0.5 \%$ EDTA chelating agent was spread over the cornea and the calcific deposits were scraped under aseptic precautions (Figure 3). The EDTA solution was slightly warmed prior to application and the superficial corneal epithelium was removed in order to prevent recurrence.

The chelated and scraped deposits were sent for histopathological examination. Haematoxylin and Eosin stained slide showed dystrophic calcium deposits along with stippled basophilia merging to form an array in the Bowman's layer.

The anterior segment optical coherence tomography scan showed hyper reflectivity in the corneal epithelium basement membrane, Bowman's layer and superficial stromal layers due to the deposition of calcium phosphate granules, traces of sulphur and silicone.

The scan was repeated one month after the chelation procedure and the reduction in the hyper reflectivity and healing of corneal epithelium was noticed.
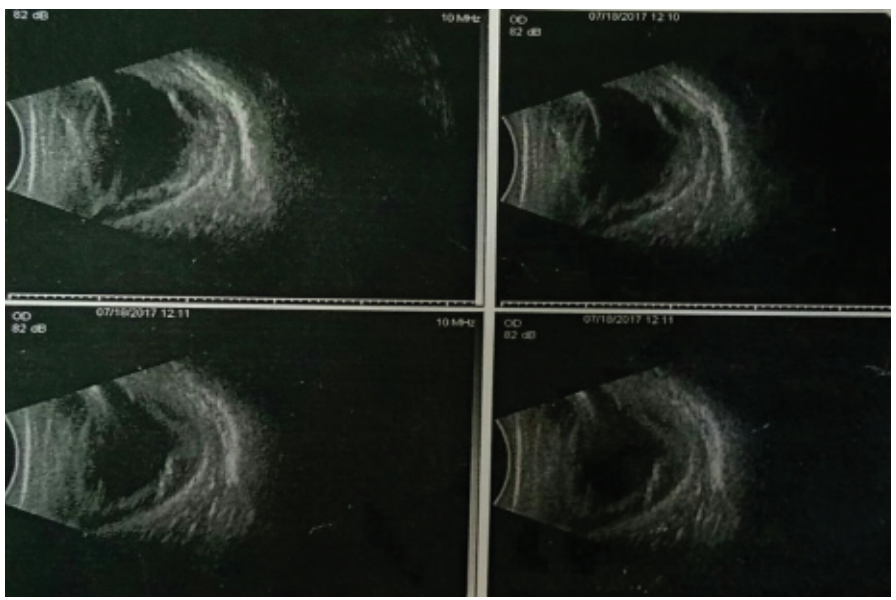

Figure 1: Indirect ophthalmoscopy and B scan revealed a total, funnel shaped rhegmatogenous retinal detachment in the right eye.

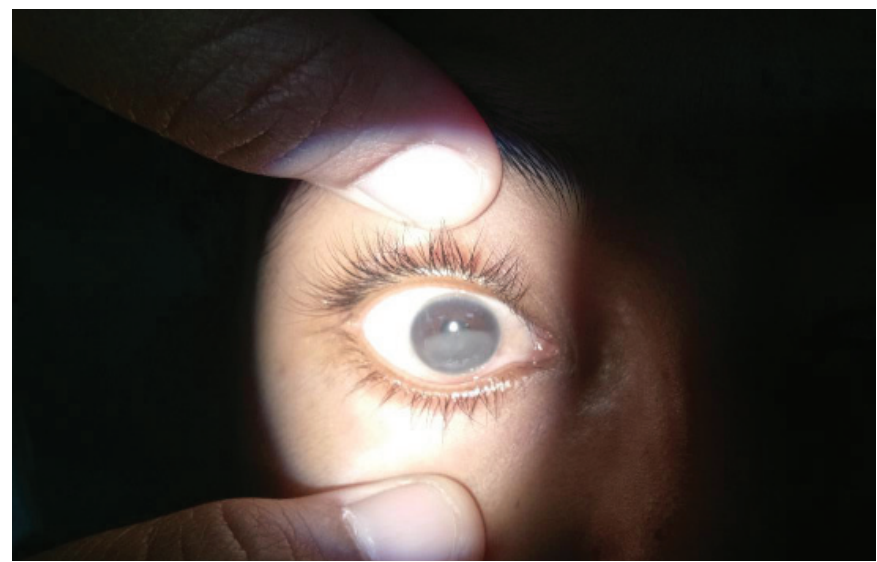

Figure 2: Shows horizontal band shaped keratopathy covering the inferior quadrant of the cornea which is encroaching the pupillary area.

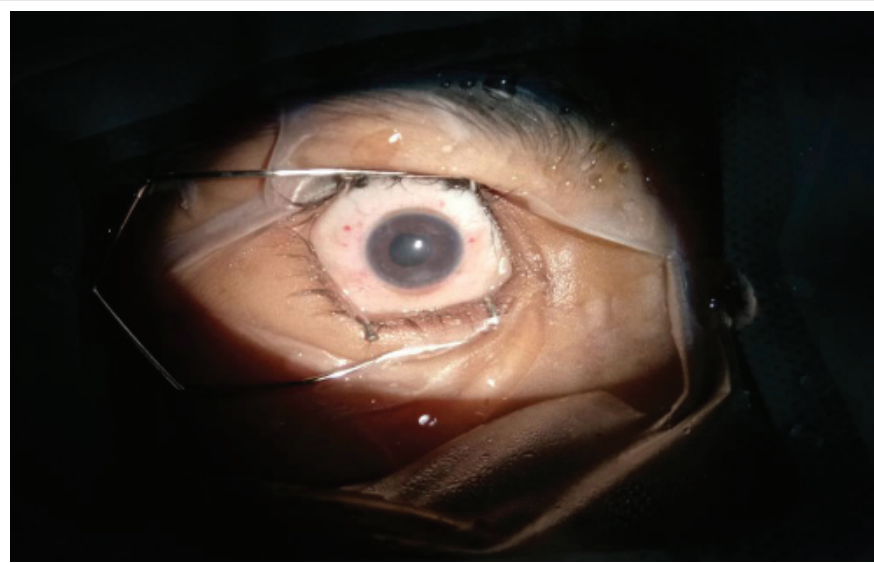

Figure 2: Shows the clear cornea after application of a neutral, autoclaved solution of $0.5 \%$ EDTA chelating agent and scraping of the calcific deposits under aseptic precautions.

Two weeks after the laser was performed in the right eye, silicon oil was removed and secondary IOL implantation was done. The patients vision improved to counting fingers at 4 metres.

The left eye lensectomy with anterior vitrectomy along with secondary IOL implantation was done one month later and the post-operative vision in the left eye improved to $6 / 24$. 


\section{Discussion}

Silicone oil acts as an effective internal tamponade agent. Due to its unique property of high surface tension, it helps to displace the detached retina posteriorly to help in its reattachment. This also helps in preventing complications associated with the condition like proliferative vitreoretinopathy. Polydimethylsiloxane has a less viscosity than the vitreous humour and therefore helps in reattachment of superior retinal breaks or tears. High density silicon oils, which are mixtures of polydimethylsiloxane and semi fluorinated alkanes have a higher viscosity. As these tend to settle at the bottom of the vitreous cavity, they are useful in treating inferior detachments and tears of the retina ${ }^{4}$. A mixture of both silicon oils can accelerate side effects associated with the usage of these oils than when used individually. The most common side effect being band shaped keratopathy as was seen in our case [4]. Moreover, the removal of the injected silicon oil must be planned appropriately to prevent further emulsification of the oils contained in the mixture. Due to the lack of resources and the poor socioeconomic status of our patient, she was lost to follow-up and the silicon oil was not removed at the right time. Aphakia was another aggravating factor in our case as the injected silicon oil bubble expanded to a much larger extent than in normal patients due to the larger anterior chamber depth. This damaged the corneal endothelium and stromal layers leading to the deposition of calcific deposits in the stroma, Bowman's and epithelial layers. Band shaped keratopathy is characterised by chronic deposition of calcium hydroxyapatite salts in the Bowman's layer, in the epithelial basement membrane and anterior stromal lamellae of corneal epithelium due to a lower metabolic rate of the cornea resulting in less metabolite production [5,6]. The carbon dioxide evaporation on the other hand, remains unchanged, thereby causing an increase in the $\mathrm{pH}$ due to the excess accumulation of lactic acid thereby causing precipitation. This in addition, may be associated with an abnormality and irregularity in the transport and mobilisation of calcium in the interstitial fluid or an abnormal functioning in the sodium bicarbonate transporter (NBCe1) [7]. In addition, in cases of bilateral congenital cataracts, the immediate postoperative rehabilitation with correction of aphakia and secondary IOL implantation gives a more predictable refraction postoperatively with minimal complications [8-10].

\section{Conclusion}

Band shaped keratopathy secondary to silicone oil injection in an aphakic eye post vitreoretinal surgery is a rare complication. Based on the depth of involvement of layers of the cornea, a suitable treatment protocol must be implemented. Although EDTA chelation is the first line of treatment, keratoplasty is preferred in some cases with deeper involvement who have a deep anterior chamber and larger amounts of silicone oil pushing against the corneal layers and causing erosion and deposition of residual calcific deposits in the corneal layers. ${ }^{1}$

\section{Acknowledgement}

Dr. Seema Channabasappa for performing the surgery and Dr. Nischala Balakrishna for surgical assistance and for compilation of data.

\section{References}

1. Cibis PA, Becker B, Okun E, Canaan S (1962) The use of liquid silicone in retinal detachment surgery. Arch Ophthalmol 68: 590-599. Link: https://bit.ly/3ehaN4

2. Beekhuis WH, Van RIJ G, Zinojnovic R (1985) Silicon oil keratopathy: Indications for keratoplasty. Br J Ophthalmol 69: 247-253. Link: https://bit.ly/2XwEzfz

3. Reim M, Schutte E, Cattepoel H (1971) Experimentelle Variation der Spiegel einiger Metabolite in der Cornea. Ber Dtsch Ophthalmol Ges 71: 84-87. Link: https://bit.ly/2Vljlc3

4. Anjan Nan (2015) A worldwide yearly survey of new data in adverse drug reactions; in Side Effects of Drugs Annual.

5. Pouliquen Y, Haye C, Bisson J, Offret G (1967) Ultrastructure de la keratopathie en bandelette. Arch Ophtalmol 27: 149-158. Link: https://bit.ly/3c5ET8W

6. O'Connor GR (1972) Calcific band keratopathy. Trans Am Ophthalmol Soc 70: 58-81. Link: https://bit.ly/2UWlyBq

7. (2016) Band Shaped Keratopathy: Symptoms, Causes and Management American International Medical University. Link: https://bit.ly/2Xo3UYL

8. Speeg-Schatz C, Flament J, Weissrock M (2005) Congenital Cataract Extraction wirh primary aphakia and secondary intraocular lens implantation in the ciliary sulcus. J Catarct Refract Surg 31: 750-756. Link: https://bit.ly/3e4zErQ

9. Kim DH, Kim JH, Kim SJ, Yu YS (2012) Long term results of Bilateral Cingenutal Catarct Surgery,Aphakic Glasses and Secondary IOL Implantation. Acta Ophthlmol 90: 231-236. Link: https://bit.ly/2VlhrOv

10. Autrata R, Rehurek J, Vodicková K (2005) Visual results after primary intraocular lens implantation or contact lens correction for aphakia in the first year of age. Ophthalmologica. 219: 72-79. Link: https://bit.ly/39XewAz 